\title{
GLOBAL REGULARITY ON 3-DIMENSIONAL SOLVMANIFOLDS
}

\author{
JACEK M. CYGAN AND LEONARD F. RICHARDSON
}

\begin{abstract}
Let $M$ be any 3-dimensional (nonabelian) compact solvmanifold. We apply the methods of representation theory to study the convergence of Fourier series of smooth global solutions to first order invariant partial differential equations $D f=g$ in $C^{\infty}(M)$. We show that smooth infinite-dimensional irreducible solutions, when they exist, satisfy estimates strong enough to guarantee uniform convergence of the irreducible (or primary) Fourier series to a smooth global solution.
\end{abstract}

\section{INTRODUCTION}

Let $S$ be a solvable Lie group, and $\Gamma$ a discrete subgroup of $S$ with compact quotient $\Gamma \backslash S$. There is then a unique probability measure $\nu$ on $\Gamma \backslash S$ that is invariant under translation on the right by elements of $S$. The regular representation of $S$ on $L^{2}(\Gamma \backslash S, \nu)$ decomposes into a direct sum of a countable number of irreducible unitary representations $\pi$ of $S$, each of finite multiplicity $m_{\pi}$ [G]. Let $D$ be a first order differential operator with complex coefficients, leftinvariant on $S$ and viewed on $\Gamma \backslash S$. Let $(\Gamma \backslash S)^{\wedge}$ denote the dual object of $\Gamma \backslash S$. If $g \in C^{\infty}(\Gamma \backslash S)$ and if $g_{\pi}$ is an orthogonal component of $g$ corresponding to some irreducible unitary representation $\pi$, then $g_{\pi} \in C^{\infty}(\Gamma \backslash S)$ too [A-B]. Modulo unitary equivalence, we may think of $g_{\pi}$ as being a $C^{\infty}$-vector in any concrete realization, or model, of $\pi$. We are interested in algebraically well-defined conditions on $D$ under which the global solvability of $D f=g$ in $C^{\infty}(\Gamma \backslash S)$ is equivalent to the solvability of $\pi(D) f_{\pi}=g_{\pi}$ in the $C^{\infty}$-vectors for each $\pi$ in the spectrum of $\Gamma \backslash S$. In a sense, we are looking for algebraic conditions on $D$ for the reduction of a global (geometrical) problem on $\Gamma \backslash S$ to a collection of purely group (representation) theoretic problems, none of which needs to be regarded as living on the manifold $\Gamma \backslash S$. Informally speaking, operators $D$ admitting such a reduction are called globally regular (Definition $(1.1))$.

In order to describe the results, we will recall the classical situation on a torus $T^{2}$ of two dimensions (the situation being similar for $T^{n}$ with $n>2$ ). Let $D=\alpha \partial / \partial x+\beta \partial / \partial y$ and suppose for simplicity that $\alpha$ and $\beta$ are real.

Received by the editors March 16, 1990.

1980 Mathematics Subject Classification (1985 Revision). Primary 22E27, 22E30; Secondary $22 \mathrm{E} 25$.

First author supported in part by Louisiana Education Quality Support Fund contract number 86-LBR-016-04. 
Then $D$ is globally regular if and only if $\beta / \alpha$ is not a (transcendental) Liouville number. The problem with Liouville numbers is that, in solving for the Fourier transform of the solution function, small divisors occur. Now, every solvmanifold $\Gamma \backslash S$ contains the structure of a torus $T=\Gamma[S, S] \backslash S$ of dimension $\geq 1$, although this torus does not reflect any of the nonabelian structure of $S$. The only representations in $(\Gamma \backslash S)^{\wedge}$ which are not infinite dimensional are the one-dimensional characters of $\Gamma[S, S] \backslash S$. Since the presence of this torus is inescapable, we denote, for each $g \in C^{\infty}(\Gamma \backslash S)$, the sum of the one-dimensional components of $g$ by $g_{0}$. Then global regularity is defined as follows. Let $L^{2}(\Gamma \backslash S)=\bigoplus_{\pi \in(\Gamma \backslash S)^{r}, j=1, \ldots, m_{\pi}} H_{\pi, j}$ be any (noncanonical) irreducible decomposition of $L^{2}(\Gamma \backslash S)$.

(1.1) Definition. A left-invariant differential operator $D$ on $\Gamma \backslash S$ is called globally regular if the three conditions

(1) $g \in C^{\infty}(\Gamma \backslash S)$,

(2) For each $\pi \in(\Gamma \backslash S)^{\wedge}$ and $\forall j=1, \ldots, m_{\pi} \exists$ a solution in $C^{\infty}(\Gamma \backslash S)$ to $D f_{\pi, j}=g_{\pi, j}$ (where $g_{\pi, j}=(\pi, j)$-component of $g$ ), and

(3) $\exists f_{0} \in C^{\infty}(T)$ such that $D f_{0}=g_{0}$,

imply that there exists a solution in $C^{\infty}(\Gamma \backslash S)$ to $D f=g$.

Note that the solutions in (2) could be found in any convenient realization of $\pi$.

In previous papers we have dealt with nilpotent $S$. On the simplest nilmanifolds, the 3-dimensional Heisenberg manifolds, every first order differential operator $D$ in the complexified Lie algebra is globally regular [R2]. On more complicated nilmanifolds the problem of small divisors arises in the representation spaces of the group as well as on the associated torus. Moreover, if $D=X+i Y$ is regular, both $a d_{X}$ and $a d_{Y}$ must map each step of the lower central series of the Lie algebra of $S$ (nilpotent) onto a sufficiently large subset of the next step. The details are explained in [C-R1, p. 349]. The purpose of this paper is to investigate the global regularity of first order differential operators on 3-dimensional compact solvmanifolds. We show that, as in the case of the simplest nilmanifolds, every first order differential operator on a 3-dimensional compact solvmanifold is globally regular.

\section{3-DIMENSIONAL SOLVMANIFOLDS}

All 3-dimensional compact solvmanifolds can be described (up to homeomorphism) as quotients of two groups $S_{h}$ and $S_{r}$ by their various cocompact discrete subgroups. The groups $S_{h}$ and $S_{r}$ can both be described as $R^{2} \rtimes R^{1}$, where $(x, t)\left(x^{\prime}, t^{\prime}\right)=\left(x+A^{t} x^{\prime}, t+t^{\prime}\right)$. Here $A^{t}$ is a 1-parameter subgroup of $S L(2, R)$ through a matrix $A \in S L(2, Z) . S_{h}$ arises when the eigenvalues of $A$ are $\lambda>1$ and $\lambda^{-1}$, so that the orbits of $R^{1}$ in $R^{2}$ are hyperbolic. $S_{r}$ arises when $A^{t}$ is a compact group of rotations of $R^{2}$. Let $N:=R^{2} \times\{0\}$, the (abelian) nilradical of $S$. The cocompact discrete subgroups $\Gamma$ are described in [A-G-H], based upon the facts (due to Mostow) that $\Gamma \cap N$ is a discrete lattice in $R^{2}$, and that the image of $\Gamma$ under the natural projection $S \rightarrow S / N$ is a discrete lattice in $R^{1}$. We remark that $\Gamma \backslash S_{h}$ is determined up to homeomorphism by the eigenvalue $\lambda>1$ of $A$, and $\lambda$ must be such that $\lambda+\lambda^{-1} \in Z$. For this reason, we denote the 'hyperbolic' manifolds $\Gamma_{\lambda} \backslash S_{h}$. Note however that 
$S_{h}$ is independent of the value of $\lambda>1$. We have good use for the following lemma ((3.4) in [C-R2]).

(2.1) Lemma. If $S_{h}=R^{2} \rtimes R^{1}$ with the diagonalized matrix $A=\left(\begin{array}{cc}\lambda & 0 \\ 0 & \lambda^{-1}\end{array}\right)$, $\lambda>1$, and if $\Gamma_{\lambda}$ is a cocompact discrete subgroup of $S_{h}$, then $\Gamma_{\lambda} \cap N$ is an abelian lattice $\mathscr{L}$ of points $(\alpha, \beta)$ having the property that the product $\alpha \beta$ is bounded away from zero, except of course at the identity.

We need also the following

(2.2) Corollary. In the setting of the lemma above, the dual lattice $\mathscr{L}^{*}=$ $\left\{\chi_{(a, b)}: \mathscr{L} \rightarrow 1\right\}$ is also a lattice of points $(a, b)$ such that the product ab is bounded away from 0 (except of course for $(a, b)=(0,0)$ ).

This corollary will give useful information about $(\Gamma \backslash S)_{\infty}$, the infinite dimensional representations in the spectrum of $\Gamma \backslash S$, in the hyperbolic case. For all 3-dimensional compact solvmanifolds, $(\Gamma \backslash S)_{\infty}$ is constructed as follows. Let $\chi_{(\alpha, \beta)} \in \hat{N}$, where $\chi_{(\alpha, \beta)}: \Gamma \cap N \rightarrow 1$. Now let $M$ be the extension of $N$ by the stabilizer of $\chi_{(\alpha, \beta)}$ in $S$, and extend $\chi$ to $M$, so $\pi_{\alpha \beta}:=\operatorname{Ind}_{M}^{S}\left(\chi_{(\alpha, \beta)}\right) \in \hat{S}$. If $S=S_{h}$ then $M=N$ and $M \backslash S \cong R$, whereas if $S=S_{r}$ then $M \cong N \times Z$ and $M \backslash S \cong R / Z=$ the circle group. If $H_{(\chi, M)}$ is the standard Mackey induced representation space, then $H_{(\chi, M)}=\{f: S \rightarrow$ $\left.C\left|f(m s)=\chi_{(\alpha, \beta)}(m) f(s),\right| f \mid \in L^{2}(M \backslash S)\right\}$. Define $L: H_{(\chi, M)} \rightarrow L^{2}(\Gamma \backslash S)$ by $(L f)(\Gamma s)=\sum_{\Gamma \cap M \backslash \Gamma} f(\gamma s)$. Then $L$ is a right $S$-invariant injection. If $\operatorname{Int}(\chi, M)=\left\{t \in R \mid \chi^{\exp t T}: \Gamma \cap M \rightarrow 1\right\}$ where $\chi^{a}(b)=\chi\left(a^{-1} b a\right)$ then the multiplicity of $\pi_{\alpha \beta}$ in $L^{2}(\Gamma \backslash S)$ equals the number of distinct $\Gamma$-orbits in $\operatorname{Int}(\chi, M)$.

In the case of $S=S_{h}$, it is easiest to describe $\Gamma_{\lambda} \backslash S_{h}$ if we take $\Gamma_{\lambda} \cap$ $N=Z^{2}$ and $A \in S L(2, Z)$. However, the model for $\pi_{\alpha \beta}$ is simplest if $A$ is diagonalized $\left(A=\left(\begin{array}{cc}\lambda & 0 \\ 0 & \lambda^{-1}\end{array}\right)\right.$ as in Lemma (2.1)) and then $\Gamma \cap N$ is more difficult to describe. Nevertheless, the Corollary (2.2) above shows that in this model $\left\{\pi_{\alpha \beta} \in\left(\Gamma_{\lambda} \backslash S_{h}\right)_{\infty}\right\}$ has $\alpha \beta$ bounded away from zero. We remark upon the fact that $\alpha \beta \neq 0$ prevents $\pi_{\alpha \beta}$ from being a representation of the (less well-behaved) ' $a x+b$ ' group which is a quotient of $S_{h}$.

We need to say a few words about the rotational three dimensional solvmanifolds $\Gamma \backslash S_{r}$ as well. Unlike the hyperbolic case, there are only finitely many $\Gamma \backslash S_{r}$ up to homeomorphism. We take $A \in S L(2, Z)$, but this time with no eigenvalues $>1$. Now $A$ turns out to be similar to a rotation by $2 \pi / p$, where $p=1,2,3,4$, or 6 (see [A-G-H]). Since $S_{r}$ is independent of $p$ (up to group isomorphism), we denote the distinct rotational three dimensional solvmanifolds by $\Gamma_{p} \backslash S_{r}, p=1,2,3,4$, or 6 .

Our main result is

(2.3) Theorem. Let $\Gamma \backslash S$ be any nonabelian 3-dimensional compact solvmanifold. If $D \in \mathscr{S}^{C}$, the complexified Lie algebra of $S$, then $D$ is globally regular on $\Gamma \backslash S$.

Since this result has been proved in an earlier paper when $S$ is nilpotent, we concern ourselves here only with the manifolds $\Gamma_{\lambda} \backslash S_{h}$ and $\Gamma_{p} \backslash S_{r}$. Note also that the associated torus $T$ is 1-dimensional, so that small divisors cannot 
occur on $T$. Thus condition (3) of Definition (1.1) will be satisfied automatically whenever (2) holds.

We will divide our proof of the theorem into two sections, one dealing with the rotational group $S_{r}$, the other with the hyperbolic one, $S_{h}$.

\section{Proof of the Theorem-CASE of $\Gamma_{p} \backslash S_{r}$}

Let $\mathscr{S}_{r}$ be the Lie algebra of $S_{r}$ with a linear basis $T, X, Y$ and the commutation relations $[T, X]=-\frac{2 \pi}{p} Y$ and $[T, Y]=\frac{2 \pi}{p} X$. Let $\pi_{\alpha \beta}$ be a generic infinite-dimensional representation in $\left(\Gamma_{p} \backslash S_{r}\right)^{\wedge}$, acting in $L^{2}(T)$, where $T=M \backslash S_{r}$, a 1-dimensional torus as described in $\S 2$. For $f \in L^{2}(T)$ the action of $\pi_{\alpha \beta}$ on $f$ is given by

$$
\pi_{\alpha \beta}(x, y ; t) f(\tau)=\exp \left(2 \pi i\left\langle(\alpha, \beta) \sigma\left(\frac{2 \pi}{p} \tau\right),(x, y)\right\rangle\right) f(\tau+t),
$$

where $\sigma(s)=\left(\begin{array}{c}\cos s \\ \sin s \\ -\sin s \cos s\end{array}\right)$. For the basis vector fields $X, Y, T$ this amounts to

$$
\begin{aligned}
& d \pi_{\alpha \beta}(X)=2 \pi i\left(\alpha \cos \frac{2 \pi}{p} t-\beta \sin \frac{2 \pi}{p} t\right), \\
& d \pi_{\alpha \beta}(Y)=2 \pi i\left(\alpha \sin \frac{2 \pi}{p} t+\beta \cos \frac{2 \pi}{p} t\right), \\
& d \pi_{\alpha \beta}(T)=\frac{d}{d t} .
\end{aligned}
$$

Since the constant $p$ plays a negligible role in the proof (even though it classifies the rotational 3-dimensional solvmanifolds), we will set $p=1$ in what follows.

We will break the proof into two cases of $D \in \mathscr{S}_{r} C$.

Case 1. $D=X+\gamma Y, \quad \gamma \in C$. This essentially covers all $D \in \mathscr{N}^{C}$ (up to a constant factor) since $D=Y$ and $D=X$ behave alike and the case of $D=0$ is trivial.

Case 2. $D=T+i(a X+b Y), a, b \in R$. This covers all $D \in \mathscr{S}_{r}^{C} \sim \mathscr{N}^{C}$ (up to an isomorphism) because the real part of $D$ can be absorbed into $T$.

Proof of Case 1. Write $D=X+(a+i b) Y, a, b \in R$. Then, in view of (3.1), the operator $d \pi_{\alpha \beta}(D)$ is a multiplication by the function

$$
\begin{aligned}
D_{\alpha \beta}(t): & =2 \pi i[(\alpha+a \beta+i b \beta) \cos 2 \pi t+(a \alpha-\beta+i b \alpha) \sin 2 \pi t] \\
& =2 \pi i(w \cos 2 \pi t+z \sin 2 \pi t) .
\end{aligned}
$$

By hypothesis, the equation $d \pi_{\alpha \beta}(D) f_{\alpha \beta}=g_{\alpha \beta}$ has the solution

$$
f_{\alpha \beta}(t)=g_{\alpha \beta}(t) / D_{\alpha \beta}(t) .
$$

To prove the theorem we need to show that $\sum_{(\alpha, \beta)} L f_{\alpha \beta} \in C^{\infty}(\Gamma \backslash S)$. Here $(\alpha, \beta)$ varies over a cross section of $\Gamma$-orbits, so that each infinite-dimensional primary summand will be spanned. By the Auslander-Brezin version of the Sobolev inequality it suffices to show $\sum_{(\alpha, \beta)}\left\|U L f_{\alpha \beta}\right\|^{2}<\infty$ for all $U \in$ $\mathscr{U}\left(\mathscr{S}_{r}\right)$. Since $L$ is an $S$-invariant isometry from $H_{\alpha \beta}$ into $L^{2}(\Gamma \backslash S)$, this is the same as to show $\sum_{(\alpha, \beta)}\left\|U f_{\alpha \beta}\right\|_{2}^{2}<\infty$. We begin by estimating the sum 
$\sum\left\|f_{\alpha \beta}\right\|_{2}^{2}$. The problem, of course, is that $D_{\alpha \beta}(t)$ in (3.3) can have zeros, and that even when it has no zeros, we must know how close $\left|D_{\alpha \beta}(t)\right|$ can come to 0 as $(\alpha, \beta) \rightarrow \infty$. We can write

$$
D_{\alpha \beta}(t)=\pi i\left((w-i z) e^{2 \pi i t}+(w+i z) e^{-2 \pi i t}\right)=\pi i\left(A e^{2 \pi i t}+B e^{-2 \pi i t}\right),
$$

with $A$ and $B$ defined by the last equation. The minimum of $\left|D_{\alpha \beta}(t)\right|$ occurs where $A$ and $B$ are rotated to opposite directions, and then

$$
\begin{aligned}
\operatorname{Min}\left|D_{\alpha \beta}(t)\right| & = \pm \pi(|A|-|B|) \\
& =\pi\left(\alpha^{2}+\beta^{2}\right)^{1 / 2}\left(\left(a^{2}+(1+b)^{2}\right)^{1 / 2}-\left(a^{2}+(1-b)^{2}\right)^{1 / 2}\right) \\
& =\left(\alpha^{2}+\beta^{2}\right)^{1 / 2} \cdot K \geq \sqrt{2}|\alpha \beta|^{1 / 2} \cdot K .
\end{aligned}
$$

If $b \neq 0$, the constant $K$ is $\neq 0$. Since $|\alpha \beta| \gg 0,\left|f_{\alpha \beta}(t)\right| \leq C\left|g_{\alpha \beta}(t)\right|$ with the constant $C$ independent of $(\alpha, \beta)$. Consequently, $\sum_{(\alpha, \beta)}\left\|f_{\alpha \beta}\right\|^{2} \leq$ $C \sum_{(\alpha, \beta)}\left\|g_{\alpha \beta}\right\|^{2}<\infty$ for $g \in C^{\infty}(\Gamma \backslash S)$.

If $b=0, D_{\alpha \beta}(t)$ does have one or more zeros and

$$
D_{\alpha \beta}(t)=2 \pi i((\alpha+a \beta) \cos 2 \pi t+(a \alpha-\beta) \sin 2 \pi t) .
$$

If $D_{\alpha \beta}\left(t_{0}\right)=0$, then $g_{\alpha \beta}\left(t_{0}\right)=0$ too, since $f_{\alpha \beta}$ given by (3.3) is in $C^{\infty}(T)$. The idea is to control $f_{\alpha \beta}$ inside specified intervals around each of the $t_{0}$ 's by the mean value theorem, and to use the monotonicity of $D_{\alpha \beta}$ on large exterior intervals to control $\left|f_{\alpha \beta}\right|$ by keeping $\left|D_{\alpha \beta}\right|$ big. We have

$$
D_{\alpha \beta}^{\prime}\left(t_{0}\right)=-2 \pi\left(\alpha^{2}+\beta^{2}\right)^{1 / 2}\left(1+a^{2}\right)^{1 / 2},
$$

and by the mean value theorem

$$
\left|D_{\alpha \beta}^{\prime}(t)-D_{\alpha \beta}^{\prime}\left(t_{0}\right)\right| \leq\left|t-t_{0}\right| 4 \pi^{2}\left(\alpha^{2}+\beta^{2}\right)^{1 / 2}\left(1+a^{2}\right)^{1 / 2} .
$$

Consequently, for $\left|t-t_{0}\right|<1 / 4 \pi,\left|D_{\alpha \beta}^{\prime}(t)\right| \geq \pi\left(\alpha^{2}+\beta^{2}\right)^{1 / 2}\left(1+a^{2}\right)^{1 / 2}$ and, since $D_{\alpha \beta}\left(t_{0}\right)=0$,

$$
\left|D_{\alpha \beta}(t)\right| \geq \pi\left(\alpha^{2}+\beta^{2}\right)^{1 / 2}\left(1+a^{2}\right)^{1 / 2}\left|t-t_{0}\right|
$$

again by the mean value theorem. For $g_{\alpha \beta}(t)$ we have the estimate

$$
\left|g_{\alpha \beta}(t)\right|=\left|g_{\alpha \beta}(t)-g_{\alpha \beta}\left(t_{0}\right)\right| \leq\left\|T g_{\alpha \beta}\right\|_{\infty}\left|t-t_{0}\right|,
$$

$\|\cdot\|_{\infty}$ denoting the sup norm on the torus $M \backslash S$. So

$$
\left|f_{\alpha \beta}(t)\right| \leq\left\|T g_{\alpha \beta}\right\|_{\infty} \frac{1}{\pi}\left(\alpha^{2}+\beta^{2}\right)^{-1 / 2}\left(1+a^{2}\right)^{-1 / 2}, \quad \text { for }\left|t-t_{0}\right|<1 / 4 \pi \text {. }
$$

On the intervals complementary to $\left|t-t_{0}\right|<1 / 4 \pi$,

$$
\left|D_{\alpha \beta}(t)\right| \geq\left|D_{\alpha \beta}\left(t_{0} \pm 1 / 4 \pi\right)\right| \geq \pi\left(\alpha^{2}+\beta^{2}\right)^{1 / 2}\left(1+a^{2}\right)^{1 / 2} / 4 \pi,
$$

hence

$$
\left|f_{\alpha \beta}(t)\right| \leq 4\left\|g_{\alpha \beta}\right\|_{\infty}\left(\alpha^{2}+\beta^{2}\right)^{-1 / 2}\left(1+a^{2}\right)^{-1 / 2} \text { for }\left|t-t_{0}\right| \geq 1 / 4 \pi
$$

for each of the two values $t_{0}$ of $t$ where $D_{\alpha \beta}$ vanishes. By (3.6) and (3.7), for all $t$ on the torus $M \backslash S$ we have the following estimate:

$$
\left|f_{\alpha \beta}(t)\right| \leq\left(\left\|T g_{\alpha \beta}\right\|_{\infty} / \pi+4\left\|g_{\alpha \beta}\right\|_{\infty}\right)\left(\alpha^{2}+\beta^{2}\right)^{-1 / 2}\left(1+a^{2}\right)^{-1 / 2} .
$$

By Sobolev's inequality we may replace the sup norms in (3.8) by $L^{2}$-norms of $g_{\alpha \beta}, T g_{\alpha \beta}$, and $T^{2} g_{\alpha \beta}$. The sum $\sum_{(\alpha, \beta)}\left\|f_{\alpha \beta}\right\|_{2}^{2}$ is finite because each sum $\sum_{(\alpha, \beta)}\left\|T^{k} g_{\alpha \beta}\right\|_{2}^{2}$ for $k=0,1,2$ is finite and $|\alpha \beta|$ is bounded away from zero. 
Next, we must show $\sum_{(\alpha, \beta)}\left\|U f_{\alpha \beta}\right\|_{2}^{2}<\infty$ for every fixed $U \in \mathscr{U}\left(\mathscr{S}_{r}\right)$. Since $[D, \mathscr{N}]=0$, this is true for all $U \in \mathscr{U}(\mathscr{N})$. It remains to show $\sum_{(\alpha, \beta)}\left\|T^{k} f_{\alpha \beta}\right\|_{2}^{2}<\infty$ for $k=1,2, \ldots$, because every $U \in \mathscr{U}\left(\mathscr{S}_{r}\right)$ can be written as a linear combination of monomials $T^{k} V$ with $V$ in $\mathscr{U}(\mathscr{N})$.

(3.9) Proposition. For $D=X+\gamma Y, \gamma \in C$ and $k=1,2,3, \ldots$ the $(k+1)$-fold bracket product $\left[D\left[D \cdots\left[D, T^{k}\right] \cdots\right]\right]$ is 0 .

Proof of Proposition. By Leibnitz's rule for the derivation $[D, \cdot]$ of the algebra $\mathscr{U}\left(\mathscr{S}_{r}\right)$ we have $\left[D, T^{k}\right]=\sum_{j=1}^{k} T \cdots T[D, T] T \cdots T$, with $[D, T]$ at $j$ th place. Since $D$ commutes with $\mathscr{N}$ and $[D, T]=-X+\gamma Y \in \mathscr{N}$, the derivation repeated $k+1$ times is zero.

(3.10) Proposition. For $f_{\alpha \beta}$ as in (3.3) we have

$$
T^{k} f_{\alpha \beta}=h_{k} / D_{\alpha \beta}^{k+1}
$$

with

$$
\begin{aligned}
h_{k}= & {\left[D\left[D \cdots\left[D, T^{k}\right] \cdots\right]\right] g_{\alpha \beta}+D\left[D \cdots\left[D, T^{k}\right] \cdots\right] g_{\alpha \beta} } \\
& +\cdots+D^{k-1}\left[D, T^{k}\right]+D^{k} T^{k} g_{\alpha \beta} .
\end{aligned}
$$

The first bracket involves $k \quad D$ 's with the number of $D$ 's inside the brackets decreasing by one in each successive summand.

Proof of Proposition. In view of Proposition (3.9) this is formula (1.8) on p. 353 of [C-R1].

The estimates on $T^{k} f_{\alpha \beta}$ given by (3.11) can now be done in a manner similar to that already presented. If $D_{\alpha \beta} \neq 0$, we use the inequality (3.4) raised to the power $k+1$ to estimate the denominator $\left|D_{\alpha \beta}\right|^{k+1}$. If $D_{\alpha \beta}\left(t_{0}\right)=0$, the numerator $h_{k}$ in $(3.11)$ must have a $(k+1)$ th order zero at $t_{0}$ since $T^{k} f_{\alpha \beta}$ is $C^{\infty}$. Instead of the estimate (3.5) we use

$$
\left|h_{k}(t)\right| \leq\left\|T^{k+1} h_{k}\right\|_{\infty}\left|t-t_{0}\right|^{k+1} /(k+1) !
$$

which follows from Taylor's formula. In the denominator of (3.6) and (3.7) we use the $(k+1)$ th power of the previous estimate for $D_{\alpha \beta}$.

Proof of Case 2. Here $D=T+i(a X+b Y), a, b \in R$, and

$$
\begin{aligned}
d \pi_{\alpha \beta}(D) & =\frac{d}{d t}-2 \pi((a \alpha+b \beta) \cos 2 \pi t+(b \alpha-a \beta) \sin 2 \pi t) \\
& =\frac{d}{d t}-2 \pi(A \cos 2 \pi t+B \sin 2 \pi t) .
\end{aligned}
$$

Write $A \cos 2 \pi t+B \sin 2 \pi t=\left(A^{2}+B^{2}\right)^{1 / 2} \sin 2 \pi(t+\phi)$, with the constant $\phi$ depending upon $A$ and $B$. If $d \pi_{\alpha \beta}(D) f_{\alpha \beta}=g_{\alpha \beta}$, we have

$$
\begin{aligned}
f_{\alpha \beta}(t)= & \exp \left(-2 \pi\left(A^{2}+B^{2}\right)^{1 / 2} \cos 2 \pi(t+\phi)\right) \\
& \times\left(\int_{-\phi-1 / 2}^{t} g_{\alpha \beta}(\tau) \exp \left(2 \pi\left(A^{2}+B^{2}\right)^{1 / 2} \cos 2 \pi(\tau+\phi)\right) d \tau+C\right) .
\end{aligned}
$$

Here we identify $M \backslash S=R / Z$ with the interval [ $-\phi-\frac{1}{2}, \frac{1}{2}-\phi$ ], and we use the fact that $f_{\alpha \beta}, g_{\alpha \beta}$, and the exponentials all have period 1 . Since $C$ is 
arbitrary, choosing $C=0$ and changing the variables $\tau^{\prime}=\tau+\phi, t^{\prime}=t+\phi$ we have

$$
\begin{aligned}
f_{\alpha \beta}(t-\phi) & =\int_{-1 / 2}^{t} g(\tau-\phi) \exp \left(2 \pi\left(A^{2}+B^{2}\right)^{1 / 2}(\cos 2 \pi \tau-\cos 2 \pi t)\right) d \tau \\
& =-\int_{t}^{1 / 2} \text { (same integrand as above). }
\end{aligned}
$$

The last equality follows from the periodicity of $f_{\alpha \beta}$, because $0=f_{\alpha \beta}(-1 / 2-\phi)$ $=f_{\alpha \beta}(1 / 2-\phi)$. For the estimates on $f_{\alpha \beta}(t)$ or on $f_{\alpha \beta}(t-\phi)$ we use the integral over $-1 / 2 \leq \tau \leq t$ if $-1 / 2 \leq t \leq 0$ and over $t \leq \tau \leq 1 / 2$ for $0<t<1 / 2$. That way we always have $\cos 2 \pi \tau<\cos 2 \pi t$, so that the exponent in the integral defining $f_{\alpha \beta}$ is negative. Then

$$
\left\|f_{\alpha \beta}\right\|_{\infty} \leq C\left\|g_{\alpha \beta}\right\|_{\infty} \leq C_{1}\left(\left\|g_{\alpha \beta}\right\|_{2}+\left\|T g_{\alpha \beta}\right\|_{2}\right)
$$

Since the $f_{\alpha \beta}$ 's all live on an interval of length 1 , the same inequality holds for $\left\|f_{\alpha \beta}\right\|_{2}$ with a constant, say $C_{1}^{\prime}$, independent of $(\alpha, \beta)$. Thus $\sum_{(\alpha, \beta)}\left\|f_{\alpha \beta}\right\|_{2}^{2}<$ $\infty$ and $f=\sum f_{\alpha \beta} \in L^{2}\left(\Gamma_{p} \backslash S_{r}\right)$. Next, to estimate $\sum_{(\alpha, \beta)}\left\|U f_{\alpha \beta}\right\|_{2}^{2}$ for every $U \in \mathscr{U}\left(\mathscr{S}_{r}\right)$ it suffices to consider $U=X^{k} Y^{j} T^{l}$. By (3.12) $T$ just differentiates $f_{\alpha \beta}$ yielding $2 \pi\left(A^{2}+B^{2}\right)^{1 / 2} \sin 2 \pi(t+\phi) f_{\alpha \beta}(t)+g_{\alpha \beta}(t)$ with $A, B$ depending linearly on $\alpha, \beta$. Successive powers of $T$ differentiate $g_{\alpha \beta}$ and $\sin 2 \pi(t+\phi)$, or $f_{\alpha \beta}(t)$. Operating by $X$ 's or $Y$ 's just multiplies by a polynomial in $(\alpha, \beta)$ times a (bounded) combination of sines and cosines. However, $X^{2}+Y^{2}$ acts on $L^{2}(M \backslash S)$ by multiplying by $-4 \pi^{2}\left(\alpha^{2}+\beta^{2}\right)$, so that $\sum_{(\alpha, \beta)}\left(\alpha^{2}+\beta^{2}\right)^{q}\left\|g_{\alpha \beta}\right\|_{2}^{2}<$ $\infty$. Thus $\sum_{(\alpha, \beta)}\left\|X^{k} Y^{j} T^{l} f_{\alpha \beta}\right\|_{2}^{2}$ can be estimated by $C \sum\left\|\left(X^{2}+Y^{2}\right)^{q} g_{\alpha \beta}\right\|_{2}^{2}$ for some $q$.

Remark. Although we have pretended $p=1$ in the arguments above, this was just a notational convenience, and the same arguments work just as well for general $\Gamma \backslash S_{r}$. Actually, the use of $\Gamma_{1} \backslash S_{r}$ has some independent interest however. Here, although $S_{r}$ is not nilpotent, $\Gamma \cong Z^{3}$. Thus, by the general theory of Mostow [M], $\Gamma_{1} \backslash S_{r}$ is homeomorphic to a torus $T^{3}$. Here we get a proof of global regularity for elements of $\mathscr{S}_{r}^{C}$ acting on $T^{3}$, keeping in mind that we mean global regularity with respect to the representation theory of $S_{r}$. Thus we have a natural class of operators on $T^{3}$ which are best analyzed with respect to $S_{r}$.

\section{Proof of THE THEOREM-CASE OF $\Gamma_{\lambda} \backslash S_{h}$}

Let $\mathscr{S}_{h}$ be the Lie algebra of $S_{h}$ with a linear basis $T, X, Y$ and the commutation relations $[T, X]=X \ln \lambda$ and $[T, Y]=-Y \ln \lambda$. A generic infinite dimensional representation $\pi_{\alpha \beta}$ in $\left(\Gamma_{\lambda} \backslash S_{h}\right)^{\wedge}$ acts on $L^{2}(R)$ by

$$
\pi_{\alpha \beta}(x, y, t) f(\tau)=\exp 2 \pi i\left(\alpha \lambda^{\tau} x+\beta \lambda^{-\tau} y\right) f(\tau+t) .
$$

For the basis vector fields $X, Y, T$ this amounts to

$$
d \pi_{\alpha \beta}(X)=2 \pi i \alpha \lambda^{t}, \quad d \pi_{\alpha \beta}(Y)=2 \pi i \beta \lambda^{-t}, \quad d \pi_{\alpha \beta}(T)=\frac{d}{d t} .
$$

The space $H_{\alpha \beta}^{\infty}$ of $C^{\infty}$-vectors for $\pi_{\alpha \beta}$ consists of $C^{\infty}(R)$ functions $\phi$ with

$$
\lim _{|t| \rightarrow \infty} \lambda^{m|t|} \phi^{(n)}(t)=0
$$


for every $m, n \in N$. These $\phi$ are called "super-Schwartz". We will denote this space by $\mathscr{S} \mathscr{S}(R)$.

We will break the proof into four cases of $D \in \mathscr{S}_{h} C$.

Case 1. $D=T$. Any nonzero complex multiple of $D=T+a X+b Y \in \mathscr{S}_{h}$ can be reduced up to isomorphism to $D=T$, if $a, b \in R$.

Case 2. $D=a X+b Y \in \mathscr{N} \subset \mathscr{S}_{h}, a, b \in R$.

Case 3. $D=a X+b Y \in \mathscr{N}^{C} \subset \mathscr{S}_{h}^{C}, a, b \in C$.

Case 4. $D=T+i(a X+b Y), a, b \in R$.

Proof of Case 1. $D=T$. The equation $d \pi_{\alpha \beta}(T) f_{\alpha \beta}=g_{\alpha \beta}$ becomes $(d / d t) f_{\alpha \beta}$ $=g_{\alpha \beta}$. Since both $f_{\alpha \beta}$ and $g_{\alpha \beta}$ are in $H_{\alpha \beta}^{\infty}$, we have

$$
f_{\alpha \beta}(t)=\int_{-\infty}^{t} g_{\alpha \beta}(\tau) d \tau=-\int_{t}^{\infty} g_{\alpha \beta}(\tau) d \tau .
$$

Subcase 1a. If $t \geq 0$, we use the second integral for the estimates on $f_{\alpha \beta}$ and the fact that $g_{\alpha \beta}(\tau)=X g_{\alpha \beta}(\tau) / 2 \pi i \alpha \lambda^{\tau}$, so that

$$
\left|f_{\alpha \beta}(t)\right| \leq\left\|X g_{\alpha \beta}\right\|_{\infty}(2 \pi \alpha \ln \lambda)^{-1} \lambda^{-t} \in L^{2}([0, \infty), d t) .
$$

Squaring, integrating $\int_{0}^{\infty} \cdots d t$, and applying Sobolev's inequality to estimate the norm $\left\|X g_{\alpha \beta}\right\|_{\infty}$ by a combination of $L^{2}$-norms of derivatives of $X g_{\alpha \beta}$ we obtain

$$
\left\|f_{\alpha \beta}\right\|_{L^{2}[0, \infty)}^{2} \leq \frac{c}{\alpha}\left(\left\|X g_{\alpha \beta}\right\|_{2}^{2}+\left\|T X g_{\alpha \beta}\right\|_{2}^{2}\right)
$$

for some constant $c$. As in the proof of Lemma (2.1) (see [C-R2]), we pick the representative $(\alpha, \beta)$ from the orbit corresponding to $\pi$ so that $\lambda^{-2} \leq|\beta / \alpha| \leq$ 1. Since $|\alpha \beta| \gg 0$, we must have $|\alpha| \gg 0$ too. Thus $\sum_{(\alpha, \beta)}\left\|f_{\alpha \beta}\right\|_{L^{2}[0, \infty)}^{2}<\infty$.

Subcase 1b. If $t<0$, we use the first integral formula for $f_{\alpha \beta}$ in (4.2), together with the fact that $g_{\alpha \beta}(\tau)=Y g_{\alpha \beta}(\tau) / 2 \pi i \beta \lambda^{-\tau}$. Picking $(\alpha, \beta)$ as in Subcase 1a, we insure that $|\beta| \gg 0$. Now an argument almost identical to that in Subcase la shows that $f=\sum f_{\alpha \beta} \in L^{2}(R)$.

To complete Case 1 , we need to prove that $\sum\left\|U f_{\alpha \beta}\right\|_{2}^{2}<\infty$, for each fixed $U \in \mathscr{U}\left(\mathscr{S}_{h}\right)$. If $U=T^{p}$ there is no problem, since $[D, U]=0$. If $U=$ $X^{m} Y^{n} T^{p}, U f_{\alpha \beta}(t)=(2 \pi i)^{m+n} \alpha^{m} \beta^{n} \lambda^{(m-n) t} T^{p-1} g_{\alpha \beta}(t)$. If $p \geq 1$, there is no problem. But if $p=0$, we estimate $\left|g_{\alpha \beta}(\tau)\right|$ by a $\left\|V g_{\alpha \beta}\right\| \lambda^{k t}$, where $V \in$ $\mathscr{U}\left(\mathscr{S}_{h}\right)$ and $k \in Z$ are chosen one way for $t<0$ and another way for $t \geq 0$, so as to assure that $U f_{\alpha \beta} \in L^{2}(-\infty, 0)$ and $U f_{\alpha \beta} \in L^{2}(0, \infty)$ separately. Hence $\sum U f_{\alpha \beta} \in L^{2}(R)$.

Case 2. Suppose $D=a X+b Y$, with $a$ and $b$ real. Here,

$$
f_{\alpha \beta}(t)=g_{\alpha \beta}(t) / 2 \pi i\left(a \alpha \lambda^{t}+b \beta \lambda^{-t}\right) \text {. }
$$

We will need to consider the occurrence of zeros in the denominator. Observe that the denominator vanishes if and only if $\lambda^{2 t}=-b \beta / a \alpha$, which means $t=t_{0}=\frac{1}{2} \log _{\lambda}(-b \beta / a \alpha)$.

Subcase 2a. Suppose $b \beta / a \alpha>0$, so that $h(t)=\left(a \alpha \lambda^{t}+b \beta \lambda^{-t}\right)^{-1}$ has a maximum (absolute) value on $R$. At the maximum point $h^{\prime}(t)=0$, so that 
$\lambda^{2 t}=b \beta / a \alpha$. Thus $h\left(t_{\max }\right)=\lambda^{t_{\max }} / 2 b \beta= \pm 1 / 2|a b \alpha \beta|^{1 / 2}$. Thus $\left|f_{\alpha \beta}(t)\right| \leq$ $\left|g_{\alpha \beta}(t)\right| / 2|a b \alpha \beta|^{1 / 2}$. Since $|\alpha \beta| \gg 0$, we have $\sum f_{\alpha \beta} \in L^{2}$, where the sum is over those $(\alpha, \beta)$ such that $b \beta / a \alpha>0$. The same is true for $\sum U f_{\alpha \beta}$, for each fixed $U \in \mathscr{U}\left(\mathscr{S}_{h}\right)$ since

(1) if $U \in \mathscr{U}(\mathscr{N}),[U, D]=0$, and

(2) if $U=X^{m} Y^{n} T^{k}$, then

$$
\frac{d}{d t}\left(g_{\alpha \beta}(t) h(t)\right)=g_{\alpha \beta}^{\prime} h+g_{\alpha \beta} h^{\prime}=\left(T g_{\alpha \beta}\right) h+g_{\alpha \beta} h^{\prime},
$$

and $g_{\alpha \beta} h^{\prime}=(X-Y) g_{\alpha \beta} \cdot h_{\alpha \beta}^{2}$, which is still $L^{2}$-summable.

Subcase $2 \mathrm{~b}$. Either $a \alpha=0$ or $b \beta=0$. Since $|\alpha \beta| \gg 0$ (except at $(0,0)$ ) this means $a=0$ or $b=0$ (but not both). If $b=0$, then $f_{\alpha \beta}(t)=$ $-Y g_{\alpha \beta}(t) / 4 \pi^{2} a \alpha \beta$. Then $\sum f_{\alpha \beta} \in L^{2}$, since $|\alpha \beta| \gg 0$. A similar argument applies if $a=0$.

Subcase 2c. Here, we suppose there exists a zero, $t_{0}=\frac{1}{2} \log _{\lambda}(-b \beta / a \alpha)$. Then $g_{\alpha \beta}\left(t_{0}\right)=0$ too, and $f_{\alpha \beta}(t)=\left(g_{\alpha \beta}(t)-g_{\alpha \beta}\left(t_{0}\right)\right) / 2 \pi i\left(a \alpha \lambda^{t}+b \beta \lambda^{-t}\right)$. Since it would suffice to prove separately the summability of the real and imaginary parts of $f_{\alpha \beta}$, we can assume wlog that $g_{\alpha \beta}$ is real-valued. For some $\tau$ between 0 and $t$, we have $f_{\alpha \beta}(t)=g_{\alpha \beta}^{\prime}(\tau) / 2 \pi i\left(a \alpha \lambda^{\tau}-b \beta \lambda^{-\tau}\right) \ln \lambda$, by the general mean value theorem. Since $b \beta / a \alpha<0$ in this case, the new denominator cannot vanish. Hence, recalling that $\tau$ depends on $t$,

$$
\begin{aligned}
4 \pi^{2} \int_{R}\left|f_{\alpha \beta}(t)\right|^{2} d t & \\
= & \int_{\left|t-t_{0}\right| \leq 1}\left|g_{\alpha \beta}^{\prime}(\tau)\right|^{2}\left|a \alpha \lambda^{\tau}-b \beta \lambda^{-\tau}\right|^{-2}|\ln \lambda|^{-2} d t \\
& +\int_{\left|t-t_{0}\right| \geq 1}\left|g_{\alpha \beta}(t)\right|^{2}\left|a \alpha \lambda^{t}+b \beta \lambda^{-t}\right|^{-2} d t \\
\leq & \frac{\left\|g_{\alpha \beta}^{\prime}\right\|_{\infty}^{2}}{-4 a \alpha b \beta \ln ^{2} \lambda}+\int_{\left|t-t_{0}\right| \geq 1}\left|g_{\alpha \beta}(t)\right|^{2} \cdot \operatorname{Max}\left|a \alpha \lambda^{t}+b \beta \lambda^{-t}\right|^{-2} d t .
\end{aligned}
$$

The first summand is summable over $(\alpha, \beta)$, by Sobolev's inequality. However, by the mean value theorem, $\left|a \alpha \lambda^{t}+b \beta \lambda^{-t}\right|=\left|\left(a \alpha \lambda^{\tau}-b \beta \lambda^{-\tau}\right)(\ln \lambda)\left(t-t_{0}\right)\right| \geq$ $2(-a \alpha b \beta)^{1 / 2}|\ln \lambda|$, if $\left|t-t_{0}\right| \geq 1$. Thus

$$
\pi^{2} \int_{R}\left|f_{\alpha \beta}(t)\right|^{2} d t \leq \frac{\left\|g_{\alpha \beta}^{\prime}\right\|_{\infty}^{2}}{|a \alpha b \beta| \ln ^{2} \lambda}+\int_{R} \frac{\left|g_{\alpha \beta}(t)\right|^{2}}{|a \alpha b \beta| \ln ^{2} \lambda} d t,
$$

and so $\sum f_{\alpha \beta} \in L^{2}$.

If $U \in \mathscr{U}(\mathcal{N})$, then $\sum U f_{\alpha \beta} \in L^{2}$ too, since $[D, U]=0$. So it suffices to consider $\sum T^{k} f_{\alpha \beta}$, for each fixed $k \in Z^{+}$. First, consider $k=1$. $2 \pi i T f_{\alpha \beta}(t)=h(t)\left(a \alpha \lambda^{t}+b \beta \lambda^{-t}\right)^{-2}$, where $h(t)=\left(a \alpha \lambda^{t}+b \beta \lambda^{-t}\right) g_{\alpha \beta}^{\prime}(t)-$ $g_{\alpha \beta}(t)\left(a \alpha \lambda^{t}-b \beta \lambda^{-t}\right) \ln \lambda$. Here $h(t)$ must have a zero of order at least two at $t=t_{0}$. Therefore, using a Taylor remainder of degree two in $\left(t-t_{0}\right)$, there exist $\tau$ and $\tau^{\prime}$ such that $T f_{\alpha \beta}(t)=h^{\prime \prime}\left(\tau^{\prime}\right) / D^{\prime \prime}(\tau) 2 \pi i$ where $D(t)=$ $\left(a \alpha \lambda^{t}+b \beta \lambda^{-t}\right) \ln \lambda$. But

$$
\left|D^{\prime \prime}(t)\right|=2\left(\left[\left(a \alpha \lambda^{\tau}-b \beta \lambda^{-\tau}\right) \ln \lambda\right]^{2}+\left[\left(a \alpha \lambda^{\tau}+b \beta \lambda^{-\tau}\right) \ln \lambda\right]^{2}\right) \geq 8|a \alpha b \beta| \ln ^{2} \lambda,
$$


since the second square is positive. Thus $\left|T f_{\alpha \beta}(t)\right| \leq\left|h^{\prime \prime}\left(\tau^{\prime}\right)\right| / 16 \pi|a \alpha b \beta|$. Hence

$$
\begin{aligned}
4 \pi^{2} \int_{R}\left|T f_{\alpha \beta}\right|^{2} \leq & \int_{\left|t-t_{0}\right| \leq 1}\left\|h^{\prime \prime}\right\|_{\infty} / 64(a \alpha b \beta)^{2} d t \\
& +\int_{R}|h(t)|^{2} / \operatorname{Min}\left\{|D(t)|^{2}|| t-t_{0} \mid \geq 1\right\} d t .
\end{aligned}
$$

But $\left\|h^{\prime \prime}\right\|_{\infty}$ is bounded by norms of derivatives of $g_{\alpha \beta}$, providing good summability over $(\alpha, \beta)$ of the first term. Also, $|D(t)|^{2}=\left|D^{\prime \prime}(\tau)\left(t-t_{0}\right)^{2} / 2 !\right|^{2} \geq$ $16(a \alpha b \beta)^{2} \ln ^{4} \lambda \gg 0$. Thus $\sum T f_{\alpha \beta} \in L^{2}$.

Next, we consider $k>1$. We make the following observations.

$$
\left[D, T^{k}\right]=\sum_{j=1}^{k} T \cdots T[D, T] T \cdots T,
$$

where $[D, T]$ is in the $j$ th position. Hence

$$
\begin{aligned}
{\left[D,\left[D, T^{k}\right]\right] } & =\sum_{i, j=1}^{k} T \cdots T[D, T] T \cdots T \\
& =2 \sum_{1 \leq i<j \leq k} T \cdots T[D, T] T \cdots T[D, T] T \cdots T,
\end{aligned}
$$

where $[D, T]$ occupies the $i$ th and $j$ th positions. (This is a result of $\mathscr{N}$ being abelian and normal.) The $k$-fold bracket

$$
\left[D,\left[D, \ldots\left[D, T^{k}\right] \cdots\right]\right]=k ![D, T]^{k},
$$

while the $(k+1)$-fold bracket vanishes. By (1.8) of [C-R1, p.353],

$$
2 \pi i T^{k} f_{\alpha \beta}(t)=h_{k}\left(a \alpha \lambda^{t}+b \beta \lambda^{-t}\right)^{-(k+1)}
$$

where

$$
\begin{aligned}
& h_{k}=[ {\left.\left[D \cdots\left[D, T^{k}\right] \cdots\right]\right] g_{\alpha \beta}+D\left[D \cdots\left[D, T^{k}\right] \cdots\right] g_{\alpha \beta} } \\
&+\cdots+D^{k-1}\left[D, T^{k}\right] g_{\alpha \beta}+D^{k} T^{k} g_{\alpha \beta} .
\end{aligned}
$$

It follows that $\left\|T^{k} f_{\alpha \beta}\right\|_{2}^{2}=\int_{\left|t-t_{0}\right| \leq 1}+\int_{\left|t-t_{0}\right|>1}$, with the integrand being determined by (4.8).

The second integral can be estimated as in the case of $k=1$, while the denominator in the integrand in the first integral has a Taylor expansion in $\left(t-t_{0}\right)$ using derivatives of order $\leq k+1$. For $k>1$ and odd, the $(k+1)$ th derivative of $\left(a \alpha \lambda^{t}+b \beta \lambda^{-t}\right)^{k+1}$ is of the form $(d / d t)^{k+1}\left[\left(a \alpha \lambda^{t}+b \beta \lambda^{-t}\right)^{k+1}\right]$ $=\sum_{j=0}^{(k+1) / 2} c_{j}\left(a \alpha \lambda^{t}+b \beta \lambda^{-t}\right)^{k+1-2 j}\left(a \alpha \lambda^{t}-b \beta \lambda^{-t}\right)^{2 j} \ln ^{k} \lambda$ with all $c_{j} \geq 0$ and $c_{(k+1) / 2}>0$. Thus, if $k+1$ is even,

$$
\frac{d^{k+1}}{d t^{k+1}}\left(a \alpha \lambda^{t}+b \beta \lambda^{-t}\right)^{k+1} \geq C\left(a \alpha \lambda^{t}-b \beta \lambda^{-t}\right)^{k+1}, \quad \text { where } C>0 .
$$

If $k+1$ is odd, then

$$
\frac{d^{k+1}}{d t^{k+1}}\left(a \alpha \lambda^{t}+b \beta \lambda^{-t}\right)^{k+1} \geq C^{\prime}\left|a \alpha \lambda^{t}-b \beta \lambda^{-t}\right|^{k+1}, \quad \text { for } C^{\prime}>0,
$$


by a similar calculation. Hence the minima over $\left|t-t_{0}\right| \leq 1$ in the resulting estimates proceed as in the case of $k=1$.

Case 3. $D \in \mathscr{N}^{C}$. If $D \in C \cdot X$ or $D \in C \cdot Y$, then $D \in C \cdot \mathscr{N}$ and is covered by Case 2 . Otherwise, dividing by a constant, we can assume that $D=X+(a+i b) Y$, where $a, b \in R$. Thus

$$
f_{\alpha \beta}(t)=g_{\alpha \beta}(t) / 2 \pi\left(i\left(\alpha \lambda^{t}+a \beta \lambda^{-t}\right)-b \beta \lambda^{-t}\right) .
$$

Since it suffices to prove $L^{2}$-summability for the parts of $f_{\alpha \beta}$ corresponding to the real and imaginary parts of $g_{\alpha \beta}$ separately, we can assume $g_{\alpha \beta}$ is real in (4.9). Suppose also $b \neq 0$, since Case 2 would apply if $b$ were 0 . We note that $\left|f_{\alpha \beta}(t)\right| \leq\left|g_{\alpha \beta}(t) / 2 \pi b \beta \lambda^{-t}\right|$. The methods of Case 2 can be applied to prove $\sum f_{\alpha \beta} \in L^{2}$.

If $U \in \mathscr{U}(\mathscr{N})$, then $[D, U]=0$, so that $\sum U f_{\alpha \beta} \in L^{2}$. We need to prove $\sum T^{k} f_{\alpha \beta} \in L^{2}$ for each fixed $k \in N$.

We begin with $k=1$. Then $T f_{\alpha \beta}(t)$ is the derivative of the right side of (4.9). In the numerator, we get various derivatives of $g_{\alpha \beta}$, while the modulus of the denominator exceeds $4 \pi^{2} b^{2} \beta^{2} \lambda^{-2 t}$. The $\lambda^{-2 t}$ can be moved to the numerator as a derivation, and $\sum T f_{\alpha \beta} \in L^{2}$. For $k>1$, similar reasoning applies.

Case 4. $D=T+i(a X+b Y), a, b \in R$. Up to isomorphism, we could assume that $a=b=1$, except for the case in which either $a=0$ or $b=0$. Since $T+i X$ and $T+i Y$ are very similar, we need to treat only the cases $T+i X$ and $T+i(X+Y)$. Since $T+i(X+Y)$ is more complicated, we will treat this case in detail, providing brief remarks to cover the simpler case of $T+i X$.

Actually, to simplify the constants we suppose $D=T+i(X+Y) / 2 \pi$, so that

$$
f_{\alpha \beta}(t)=\left(\int_{0}^{t} g_{\alpha \beta}(x) \exp \left(-\left(\alpha \lambda^{x}+\beta \lambda^{-x}\right) / \ln \lambda\right) d x+C\right) \exp \left(\left(\alpha \lambda^{t}+\beta \lambda^{-t}\right) / \ln \lambda\right) .
$$

Subcase 4a. $\alpha>0$ and $\beta>0$. In this case $\exp \left(\left(\alpha \lambda^{t}+\beta \lambda^{-t}\right) / \ln \lambda\right) \rightarrow \infty$ as $t \rightarrow \pm \infty$ (or in case $b=0, \exp \left(\alpha \lambda^{t} / \ln \lambda\right) \rightarrow \infty$ as $t \rightarrow \infty$ and $\rightarrow 1$ as $t \rightarrow-\infty)$. Thus

$$
\begin{aligned}
f_{\alpha \beta}(t) & =-\int_{t}^{\infty} g_{\alpha \beta}(x) \exp \left(\left(-\left(\alpha \lambda^{x}+\beta \lambda^{-x}\right)+\left(\alpha \lambda^{t}+\beta \lambda^{-t}\right)\right) / \ln \lambda\right) d x \\
& =\int_{-\infty}^{t} \cdots d x .
\end{aligned}
$$

If $b=0$, the $\beta \lambda^{-x}$ and $\beta \lambda^{-t}$ terms do not appear in (4.10) and we simply use $t_{\alpha \beta}=0$ in what follows. Also, the restriction $\beta>0$ is not necessary when we deal with the $b=0$ case.

Note that $\frac{d}{d x}\left(\alpha \lambda^{x}+\beta \lambda^{-x}\right)=\left(\alpha \lambda^{x}-\beta \lambda^{-x}\right) \ln \lambda=0$ iff $x=\frac{1}{2} \log _{\lambda}(\beta / \alpha)$, which we denote henceforth by $-1 \leq t_{\alpha \beta} \leq 0$. Since $\alpha$ and $\beta>0, \alpha \lambda^{x}+\beta \lambda^{-x}$ is a decreasing function on $\left(-\infty, t_{\alpha \beta}\right)$ and an increasing function on $\left(t_{\alpha \beta}, \infty\right)$. By using the first integral in (4.10) whenever $t \geq t_{\alpha \beta}$ and the second integral whenever $t<t_{\alpha \beta}$, we can assure that the exponential function in the integrand remains bounded by 1 . In either case, $\left|g_{\alpha \beta}\right|$ can be bounded by one of its ( $X$ or $Y$ ) derivatives times an exponential function, with the result that $\sum f_{\alpha \beta} \in L^{2}$ 
of $\left(-\infty, t_{\alpha \beta}\right) \cup\left[t_{\alpha \beta}, \infty\right)=(-\infty, \infty)$. (If $b=0$, use $\left(X^{2}+Y^{2}\right) g$ instead.)

Restriction to $\lambda^{-2} \leq \beta / \alpha \leq 1$ will keep $\alpha^{-1}$ bounded in absolute value.

Next, let $U=(2 \pi)^{-n-m} X^{m} Y^{n} \in \mathscr{U}(\mathscr{N})$. If $m-n>0$ and $t>t_{\alpha \beta}$, then

$$
\left|U f_{\alpha \beta}(t)\right| \leq\left|\alpha^{m} \beta^{n} \lambda^{(m-n) t} \int_{t}^{\infty}\right| g_{\alpha \beta}(x)|d x| \leq \int_{t}^{\infty}\left|U g_{\alpha \beta}(x)\right| d x,
$$

which provides the necessary estimate. If $m-n<0$ and $t>t_{\alpha \beta}$,

$$
\left|U f_{\alpha \beta}(t)\right| \leq\left|\alpha^{m} \beta^{n}\right| \lambda^{(m-n) t} \int_{t}^{\infty}\left|g_{\alpha \beta}(x)\right| d x .
$$

But $\lambda^{-2} \leq \beta / \alpha \leq 1$ implies $t_{\alpha \beta} \geq-1$, so

$$
\begin{aligned}
\left|U f_{\alpha \beta}(t)\right| & \leq \lambda^{n-m} \int_{t}^{\infty}\left|\alpha^{m} \beta^{n} g_{\alpha \beta}(x)\right| d x \leq \lambda^{n-m} \int_{t}^{\infty}\left|\alpha^{m+n} g_{\alpha \beta}(x)\right| d x \\
& =\lambda^{n-m} \int_{t}^{\infty}\left|X^{m+n} g_{\alpha \beta}(x)\right| \lambda^{-(m+n) x}(2 \pi)^{-m-n} d x \\
& \leq \lambda^{2 n}(2 \pi)^{-m-n} \int_{t}^{\infty}\left|X^{m+n} g_{\alpha \beta}(x)\right| d x .
\end{aligned}
$$

From here the $L^{2}$-estimates proceed as earlier.

Next, suppose $m-n<0$ and $t<t_{\alpha \beta}$. Then $\lambda^{(m-n) t}>\lambda^{(m-n) t_{\alpha \beta}}$. Thus

$$
\begin{aligned}
\left|U f_{\alpha \beta}(t)\right| & \leq\left|\alpha^{m} \beta^{n}\right| \lambda^{(m-n) t} \int_{-\infty}^{t}\left|g_{\alpha \beta}(x)\right| d x \leq \int_{-\infty}^{t}\left|\alpha^{m} \beta^{n} \lambda^{(m-n) x} g_{\alpha \beta}(x)\right| d x \\
& =\int_{-\infty}^{t}\left|U g_{\alpha \beta}(x)\right| d x .
\end{aligned}
$$

The rest is as before.

Finally, for $m-n \geq 0$ and $t<t_{\alpha \beta}$, we write

$$
\begin{aligned}
\left|U f_{\alpha \beta}(t)\right| & \leq\left|\alpha^{m} \beta^{n}\right| \lambda^{(m-n) t} \int_{-\infty}^{t}\left|g_{\alpha \beta}(x)\right| d x \\
& \leq\left|\lambda^{2 m} \beta^{m} \beta^{n}\right| \lambda^{(m-n) t_{\alpha \beta}} \int_{-\infty}^{t}\left|g_{\alpha \beta}(x)\right| d x \\
& \leq \lambda^{2 m} \int_{-\infty}^{t}\left|\beta^{m+n} g_{\alpha \beta}(x)\right| d x \\
& =\int_{-\infty}^{t}\left|Y^{m+n} g_{\alpha \beta}(x)\right| \lambda^{(m+n) x} /(2 \pi)^{m+n} d x \\
& \leq(2 \pi)^{-m-n} \int_{-\infty}^{t}\left|Y^{m+n} g_{\alpha \beta}(x)\right| d x .
\end{aligned}
$$

The $L^{2}$-estimates can be completed as before.

Next, we show that $\sum_{(\alpha, \beta)} T^{k} f_{\alpha \beta} \in L^{2}$, for each $k \in N$. This follows from the next lemma.

(4.11) Lemma. Let $f$ be a solution of the equation

$$
(T+i(X+Y)) f=g .
$$


Then $T^{k} f, k=1,2,3, \ldots$, is a linear combination of monomials $X^{j} Y^{l} f$ with $j+l \leq k$ plus a linear combination of $X, Y, T$-derivatives of $g$.

Proof. We proceed by induction. For $k=1$ we have $T f=g-i X f-i Y f$. Next, $T^{k+1} f=T\left(T^{k} f\right)=T\left(X^{j} Y^{l} f\right)$ with $j+l \leq k$, where wlog we may asume $T^{k} f$ is a monomial $X^{j} Y^{l} f$.

$$
\begin{aligned}
T\left(X^{j} Y^{l} f\right)= & {\left[T, X^{j} Y^{l}\right] f+Y^{j} Y^{l} T f } \\
= & \left(\sum_{p=1}^{j} X \cdots X[T, X] X \cdots X Y^{l}+\sum_{q=1}^{l} X^{j} Y \cdots Y[T, Y] Y \cdots Y\right) f \\
& \quad+Y^{j} Y^{l} g-i X^{j+1} Y^{l} f-i X^{j} Y^{l+1} f \\
= & \left(j \ln \lambda X^{j+1} Y^{l}-l \ln \lambda X^{j} Y^{l+1}\right) f+\ldots
\end{aligned}
$$

which is the desired expression for $T^{k} f$.

Remark. Similarly, $T^{k} f$ is a linear combination of monomials $X^{j} f$ with $j \leq$ $k$ plus a linear combination of $X, T$-derivatives of $g$ if $f$ is a solution of $(T+i X) f=g$.

Subcase 4b. $\alpha<0$ and $\beta>0$. (The case $\alpha>0$ and $\beta<0$ can be treated similarly.) Once again, we have

$$
f_{\alpha \beta}(t)=\left(\int_{0}^{t} g_{\alpha \beta}(x) \exp \left(-\left(\alpha \lambda^{x}+\beta \lambda^{-x}\right) / \ln \lambda\right) d x+C\right) \exp \left(\left(\alpha \lambda^{t}+\beta \lambda^{-t}\right) / \ln \lambda\right)
$$

where the terms $\beta \lambda^{-x}$ and $\beta \lambda^{-t}$ are not present if $b=0$. Moreover, the restriction $\beta>0$ is not needed if $b=0$. We observe $\exp \left(\left(\alpha \lambda^{t}+\beta \lambda^{-t}\right) / \ln \lambda\right) \rightarrow$ 0 as $t \rightarrow+\infty$ and $\rightarrow+\infty$ as $t \rightarrow-\infty$ (or $\rightarrow 1$ as $t \rightarrow-\infty$ in case $b=0)$. In either case, since $\lim _{t \rightarrow-\infty} f_{\alpha \beta}(t)=0$,

$$
C=\int_{-\infty}^{0} g_{\alpha \beta}(x) \exp \left(-\left(\alpha \lambda^{x}+\beta \lambda^{-x}\right) / \ln \lambda\right) d x
$$

and

$$
f_{\alpha \beta}(t)=\int_{-\infty}^{t} g_{\alpha \beta}(x) e^{\psi(x, t)} d x
$$

where

$$
\psi(x, t)=\left(\alpha\left(\lambda^{t}-\lambda^{x}\right)+\beta\left(\lambda^{-t}-\lambda^{-x}\right)\right) / \ln \lambda,
$$

again with no $\beta\left(\lambda^{-t}-\lambda^{-x}\right)$ term in case $b=0$. We notice that $\psi(x, t)<0$ for $x<t$. We have the estimates

$$
\begin{aligned}
\left|f_{\alpha \beta}(t)\right| & \leq \int_{-\infty}^{t}\left|g_{\alpha \beta}(x)\right| d x \leq \int_{-\infty}^{t}\left|Y g_{\alpha \beta}(x)\right| /\left|2 \pi \beta \lambda^{-x}\right| d x \\
& \leq C \sum_{k=0}^{1}\left\|T^{k} Y g_{\alpha \beta}\right\|_{2}|\beta|^{-1} \int_{-\infty}^{t} \lambda^{x} d x \quad \text { (by Sobolev) } \\
& \leq M \sum_{k=0}^{1}\left\|T^{k} Y g_{\alpha \beta}\right\|_{2} \lambda^{t} / \ln \lambda \in L^{2}(-\infty, 0)
\end{aligned}
$$


since $|\beta|^{-1}$ is bounded. In fact, (4.13) implies that $\sum_{(\alpha, \beta)} f_{\alpha \beta} \in L^{2}(-\infty, 0)$. Next, we must consider convergence in $L^{2}(0, \infty)$. Thus for $t>0$ we write

$$
\begin{aligned}
& f_{\alpha \beta}(t)=\int_{-\infty}^{0} \cdots+\int_{0}^{t / 2} \cdots+\int_{t / 2}^{t} \cdots \\
& =\mathrm{I}_{\alpha \beta}(t) \quad+\mathrm{II}_{\alpha \beta}(t) \quad+\mathrm{III}_{\alpha \beta}(t)
\end{aligned}
$$

where the integrands are as in (4.12).

To estimate $\mathrm{I}_{\alpha \beta}$ we notice that for $x \leq 0 \leq t$ we have $\beta\left(\lambda^{-t}-\lambda^{-x}\right) \leq 0$ and $\alpha\left(\lambda^{t}-\lambda^{x}\right)<\alpha\left(\lambda^{t}-1\right)<0$. Hence

$$
\left|\mathbf{I}_{\alpha \beta}(t)\right| \leq e^{\alpha\left(\lambda^{t}-1\right) / \ln \lambda} \int_{-\infty}^{0}\left|g_{\alpha \beta}(x)\right| d x
$$

and $\sum_{(\alpha, \beta)} \mathbf{I}_{\alpha \beta} \in L^{2}(0, \infty)$. This is because $\alpha \gg 0$ makes the functions

$$
t \mapsto \exp \left(\alpha\left(\lambda^{t}-\lambda^{x}\right) / \ln \lambda\right), \quad(\alpha, \beta) \in\left(\Gamma \backslash S_{h}\right)^{\wedge},
$$

uniformly $L^{2}(0, \infty)$, while the integral $\int_{-\infty}^{0} \cdots$ in (4.14) can be estimated as in (4.13) making the sum finite.

For $\mathrm{II}_{\alpha \beta}$ we have the estimate

$$
\begin{aligned}
\left|\mathrm{II}_{\alpha \beta}(t)\right| & \leq \int_{0}^{t / 2}\left|g_{\alpha \beta}(x)\right| e^{\alpha\left(\lambda^{t}-\lambda^{x}\right) / \ln \lambda} d x \\
& \leq\left\|g_{\alpha \beta}\right\|_{\infty} \frac{t}{2} e^{\alpha\left(\lambda^{t}-\lambda^{t / 2}\right) / \ln \lambda} .
\end{aligned}
$$

The right-hand side again is $\alpha$-uniformly in $L^{2}(0, \infty)$ with $\left\|g_{\alpha \beta}\right\|_{\infty}$ being $(\alpha, \beta)$-summable.

Finally,

$$
\begin{aligned}
\left|\mathrm{III}_{\alpha \beta}(t)\right| & \leq \int_{t / 2}^{t}\left|g_{\alpha \beta}(x)\right| d x \\
& \leq \int_{t / 2}^{t}\left|X^{m} g_{\alpha \beta}(x)\right| /\left|2 \pi \alpha \lambda^{x}\right|^{m} d x \\
& \leq\left\|X^{m} g_{\alpha \beta}\right\|_{\infty} \frac{t}{2} M^{m} \lambda^{-m t},
\end{aligned}
$$

where $M$ is an upper bound on $|\alpha|^{-1}, t \lambda^{-m t} \in L^{2}(0, \infty)$, and $\left\|X_{\alpha \beta}^{m}\right\|_{\infty}^{2}$ is $(\alpha, \beta)$-summable.

Next, we must show $\sum_{(\alpha, \beta)} U f_{\alpha \beta} \in L^{2}(R)$ for every fixed $U \in \mathscr{U}\left(\mathscr{S}_{h}\right)$.

If $U=Y^{k}$ we have the estimate

$$
\left|Y^{k} f_{\alpha \beta}(t)\right| \leq \int_{-\infty}^{t}\left|Y^{k} g_{\alpha \beta}(x)\right| e^{\psi(x, t)} d x .
$$

As in the beginning of Subcase $4 \mathrm{~b}$ we can show that $\sum Y^{k} f_{\alpha \beta} \in L^{2}(R)$.

For $U=X^{k}, \sum X^{k} f_{\alpha \beta} \in L^{2}(-\infty, 0)$ because for $t \leq 0$,

$$
\left|X^{k} f_{\alpha \beta}(t)\right|=\lambda^{2 k t}|\alpha / \beta|^{k}\left|Y^{k} f_{\alpha \beta}(t)\right| \leq \lambda^{2}\left|Y^{k} f_{\alpha \beta}(t)\right|
$$

if we choose $(\alpha, \beta)$ such that $\lambda^{-2} \leq|\beta / \alpha| \leq 1$. If $t>0$, we consider $X^{k} \mathbf{I}_{\alpha \beta}$, $X^{k} \mathrm{II}_{\alpha \beta}$, and $X^{k} \mathrm{III}_{\alpha \beta}$ and we get the estimates (4.14), (4.15), and (4.16), each multiplied by $\lambda^{2 k t}$ and with $g_{\alpha \beta}$ replaced by $Y^{k} g_{\alpha \beta}$, as it was done in (4.18). 
Finally, let $U=X^{p} Y^{q} T^{r}$. Case of $r \geq 1$ reduces to $r=0$ by the Lemma (4.11). If $r=0$, we apply $X^{p}$ to $\int_{-\infty}^{t}\left|Y^{q} g_{\alpha \beta}(x)\right| e^{\psi(x, t)} d x$ as we applied $U=X^{k}$ to $\int_{-\infty}^{t} g_{\alpha \beta}(x) e^{\psi(x, t)} d x$.

Subcase 4c. $\alpha<0$ and $\beta<0$. We have

$$
f_{\alpha \beta}(t)=\left(\int_{0}^{t} g_{\alpha \beta}(x) e^{-\left(\alpha \lambda^{x}+\beta \lambda^{-x}\right) / \ln \lambda} d x+C\right) e^{\left(\alpha \lambda^{t}+\beta \lambda^{-t}\right) / \ln \lambda} .
$$

The function $C e^{\left(\alpha \lambda^{t}+\beta \lambda^{-t}\right) / \ln \lambda}$ is in $\mathscr{S S}(R)$ if $\alpha<0$ and $\beta<0$. Hence if there is a constant $C$ such that $f_{\alpha \beta}$ in (4.19) is super-Schwartz, then $f_{\alpha \beta} \in \mathscr{S} \mathscr{S}(R)$ for any fixed $C$. We will pick $C=C_{\alpha \beta}=\int_{t_{\alpha \beta}}^{0} g_{\alpha \beta}(x) e^{-\left(\alpha \lambda^{x}+\beta \lambda^{-x}\right) / \ln \lambda} d x$, where $t_{\alpha \beta}:=\frac{1}{2} \log _{\lambda}(\beta / \alpha)$, and $\alpha, \beta$ are chosen so that $\lambda^{-2}<\beta / \alpha \leq 1$. Thus we will work with

$$
f_{\alpha \beta}(t)=\left(\int_{t_{\alpha \beta}}^{t} g_{\alpha \beta}(x) e^{-\left(\alpha \lambda^{x}+\beta \lambda^{-x}\right) / \ln \lambda} d x\right) e^{\left(\alpha \lambda^{t}+\beta \lambda^{-t}\right) / \ln \lambda} .
$$

We have the estimate

$$
\begin{aligned}
&\left|f_{\alpha \beta}(t)\right| \leq e^{\left(\alpha \lambda^{t}+\beta \lambda^{-t}\right) / \ln \lambda} \int_{t_{\alpha \beta}}^{t}(2 \pi)^{-m}\left|(X+Y)^{m} g_{\alpha \beta}(x)\right| \\
& \quad \times e^{-\left(\alpha \lambda^{x}+\beta \lambda^{-x}\right) / \ln \lambda}\left(-\alpha \lambda^{x}-\beta \lambda^{-x}\right)^{-m} d x \\
& \leq e^{\cdots / \ln \lambda}\left\|(X+Y)^{m} g_{\alpha \beta}\right\|_{\infty} e^{-\cdots / \ln \lambda}(-2 \pi \cdots)^{-m}\left|t-t_{\alpha \beta}\right| \\
& \leq c \sum_{l=0}^{1}\left\|T^{l}(X+Y)^{m} g_{\alpha \beta}\right\|_{2}\left(-\alpha \lambda^{t}-\beta \lambda^{-t}\right)^{-m}\left|t-t_{\alpha \beta}\right|
\end{aligned}
$$

where $\cdots$ stands for $\alpha \lambda^{t}+\beta \lambda^{-t}$. We write the inequality (4.20) for $(\alpha, \beta)$ such that

$$
2(\alpha \beta)^{1 / 2} / \ln \lambda>m,
$$

because then the function $u \mapsto e^{-\left(\alpha u+\beta u^{-1}\right) / \ln \lambda}\left(-\alpha u-\beta u^{-1}\right)^{-m}$ with $u=\lambda^{t}$ is increasing for $t \geq t_{\alpha \beta}$ and decreasing for $t \leq t_{\alpha \beta}$. (4.21) is valid for all but a finite number of $(\alpha, \beta) \in\left(\Gamma \backslash S_{h}\right)_{\infty}^{\infty}$. Similarly, for $Y^{l} X^{k} f_{\alpha \beta}$ we have the estimate

$$
\left|Y^{l} X^{k} f_{\alpha \beta}(t)\right| \leq c_{1} \sum_{p=0}^{1}\left\|T^{p}(X+Y)^{m} g_{\alpha \beta}\right\|_{2}\left|\beta \lambda^{-t}\right|^{l}\left|\alpha \lambda^{t}\right|^{k}\left(-\alpha \lambda^{t}-\beta \lambda^{-t}\right)^{-m}\left|t-t_{\alpha \beta}\right| .
$$

But for $t \geq t_{\alpha \beta}$, if $m>k+l$

$$
\begin{aligned}
& \left|\beta \lambda^{-t}\right|^{l}\left|\alpha \lambda^{t}\right|^{k}\left(-\alpha \lambda^{t}-\beta \lambda^{-t}\right)^{-m}\left|t-t_{\alpha \beta}\right| \leq(\beta / \alpha)^{l}(-\alpha)^{k+l-m} \lambda^{t(k-l-m)}\left|t-t_{\alpha \beta}\right| \\
& \quad \leq M^{m-k-l}\left|t-t_{\alpha \beta}\right| \lambda^{t(k-l-m)} \in L^{2}(0, \infty)
\end{aligned}
$$

since $\beta / \alpha \leq 1$ and $\alpha \gg 0$.

Similarly, for $t \leq t_{\alpha \beta}$

$$
\begin{aligned}
& \left|\beta \lambda^{-t}\right|^{l}\left|\alpha \lambda^{t}\right|^{k}\left(-\alpha \lambda^{t}-\beta \lambda^{-t}\right)^{-m}\left|t-t_{\alpha \beta}\right| \leq(\alpha / \beta)^{k}(-\beta)^{k+l-m} \lambda^{t(m-l+k)}\left|t_{\alpha \beta}-t\right| \\
& \quad \leq \lambda^{2 k} M^{m-k-l}\left|t-t_{\alpha \beta}\right| \lambda^{t(m-l+k)} \in L^{2}(-\infty, 0)
\end{aligned}
$$


since $\lambda^{-2} \leq \beta / \alpha$ and $\beta \gg 0$. Thus $\sum Y^{l} X^{k} f_{\alpha \beta} \in L^{2}(R)$. Finally,

$$
\sum Y^{l} X^{k} T^{m} f_{\alpha \beta} \in L^{2}(R)
$$

by Lemma (4.11).

\section{BIBLIOGRAPHY}

[A-B] L. Auslander and J. Brezin, Uniform distribution in solvmanifolds, Adv. in Math. 7 (1971), 111-144.

[A-G-H] L. Auslander, L. Green, and F. Hahn, Flows on homogeneous spaces, Ann. of Math. Studies No. 53, Princeton Univ. Press, 1963.

[Brl] J. Brezin, Function theory on metabelian solvmanifolds, J. Funct. Anal., 10 (1972), 33-51.

[Br2] - Harmonic analysis on compact solvmanifolds, Lecture Notes in Math., vol. 602, Springer-Verlag, Berlin-Heidelberg-New York, 1977.

[C-R1] J. Cygan and L. Richardson, Global solvability on compact nilmanifolds of three or more steps, Trans. Amer. Math. Soc. 301 (1987), 343-373.

[C-R2] _ D-harmonic distributions and global hypoellipticity on nilmanifolds, Pacific J. Math. 147 (1991), 29-46.

[G] I. M. Gelfand, M. F. Graev, and I. J. Piatetskii-Shapiro, Representation theory and automorphic functions, Saunders, Philadelphia, Pa., 1969.

[G-W] S. Greenfield and N. Wallach, Remarks on global hypoellipticity, Trans. Amer. Math. Soc. 183 (1973), 153-164.

[H] R. Howe, On Frobenius reciprocity for unipotent algebraic groups over $Q$, Amer. J. Math. 93 (1971), 163-172.

[K] A. A. Kirillov, Unitary representations of nilpotent Lie groups, Uspehi Mat. Nauk 17 (1962), 57-110; English transl., Russian Math. Surveys 17 (1962), 53-104.

[M] G. D. Mostow, Homogeneous spaces with finite invariant measures, Ann. of Math. 75 (1962), 17-38.

[R1] L. Richardson, Decomposition of the $L^{2}$-space of a general compact nilmanifold, Amer. J. Math. 93 (1971), 173-190.

[R2] Global solvability on compact Heisenberg manifolds, Trans. Amer. Math. Soc. 273 (1982), 309-317.

[S] L. Schwartz, Théorie des distributions, Hermann, Paris, 1966.

[W] G. Warner, Harmonic analysis on semi-simple Lie groups I, Springer-Verlag, New York, 1972.

Department of Mathematics, Louisiana State University, Baton Rouge, Louisiana 70803 